\title{
Local recurrence of T1a rectal cancer following radical endoscopic mucosal resection: A case report
}

\author{
KEITA MATSUMOTO $^{1}$, NOBUHISA MATSUHASHI ${ }^{1}$, TAKAO TAKAHASHI ${ }^{1}$, \\ TOSHIYUKI TANAHASHI ${ }^{1}$, SATOSHI MATSUI ${ }^{1}$, TOMONARI SUETSUGU ${ }^{1}$, JESSE YU TAJIMA $^{1}$, \\ TAKEHARU IMAI ${ }^{1}$, HISASHI IMAI ${ }^{1}$, YOSHIHIRO TANAKA ${ }^{1}$, KAZUYA YAMAGUCHI $^{1}$, \\ NATSUKO SUZUI $^{2}$, TATSUHIKO MIYAZAKI ${ }^{2}$ and KAZUHIRO YOSHIDA ${ }^{1}$ \\ ${ }^{1}$ Department of Surgical Oncology and ${ }^{2}$ Pathology Division, Gifu University School of Medicine, Gifu, Gifu 501-1194, Japan
}

Received April 8, 2018; Accepted July 3, 2018

DOI: $10.3892 / \mathrm{mco} .2018 .1667$

\begin{abstract}
We herein report a case of local recurrence of Tla rectal cancer following radical endoscopic mucosal resection (EMR). A 63-year-old man underwent EMR for a 0-IIa lesion of the Ra portion of the rectum. The findings on pathological examination were tub1, T1a (SM1, $420 \mu \mathrm{m})$, ly0 and v0, and the EMR had been considered a transitional procedure. Colonoscopy performed 26 months after EMR revealed a submucosal tumor (SMT) near the EMR scar in the left wall of the Ra portion of the rectum. An abdominal enhanced computed tomography scan revealed infiltration of the thickness of the wall with limited extramural extension, and a lymph node $10 \mathrm{~mm}$ in diameter. Endoscopic ultrasound-guided fineneedle aspiration also indicated a SMT on the left side of the Ra portion of the rectum that extended from the submucosal layer to beyond the serosal layer, and a lymph node sized $17 \times 11 \mathrm{~mm}$ to the left of the Ra portion near the oral side $2 \mathrm{~cm}$ from the SMT. The pathological findings confirmed the SMT to be an adenocarcinoma with a metastatic lymph node. Local and lymph node recurrence of rectal cancer following radical EMR was diagnosed, and laparoscopic ultra-low anterior resection, D3 lymph node dissection and a diverting ileostomy were performed. The pathological findings of the excised specimen were T3 (A/SS), ly0, v3, PN1b, pPM0, pDM0, pRM0 $(100 \mu \mathrm{m})$ and $\mathrm{pN} 0(0 / 15)$. XELOX therapy was administered for 6 months postoperatively as adjuvant chemotherapy, and there has been no recurrence during the first 12 months of follow-up.
\end{abstract}

Correspondence to: Dr Nobuhisa Matsuhashi, Department of Surgical Oncology, Gifu University School of Medicine, 1-1 Yanagido, Gifu, Gifu 501-1194, Japan

E-mail: nobuhisa517@hotmail.com

Key words: T1a rectal cancer, radical endoscopic mucosal resection, local recurrence

\section{Introduction}

In recent years, endoscopic mucosal resection (EMR) has been indicated for the treatment of superficial, early-stage colorectal cancer due to its minimal invasiveness and excellent results in terms of clinical outcome $(1,2)$. In addition, taking into consideration the possibility of incomplete tumor removal due to the difficulty in resecting lesions sized $>2 \mathrm{~cm}$ by EMR, endoscopic submucosal dissection has been adapted for use in this procedure $(1,3)$. Cases of local recurrence following radical EMR are extremely rare. We herein report an extremely rare case of recurrence at 26 months following lesion removal by a curative endoscopic procedure performed according to the 2016 Colon Cancer Treatment Guidelines of the Japanese Society for Cancer of the Colon and Rectum (JSCCR guidelines) for the treatment of colorectal cancer.

\section{Case report}

A 63-year-old man underwent EMR for a 0-IIa lesion in the Ra portion of the rectum in October, 2014. The pathological findings (Fig. 1) were tub1, T1a (SM1, $420 \mu \mathrm{m}$ ), ly0 and v0, and the EMR was considered to be a transitional procedure. A colonoscopy (Fig. 2) performed 26 months after the radical EMR revealed a submucosal tumor (SMT) near the EMR scar in the rectum. The patient was referred for treatment according to the recommendations of our hospital in December, 2016.

Hematological examination revealed mild anemia. The tumor marker levels had been normal since the initial EMR treatment. An abdominal enhanced computed tomography scan (Fig. 3A and B) revealed infiltration of the thickness of the left wall of the Ra portion of the rectum with limited extramural extension, and a lymph node $10 \mathrm{~mm}$ in diameter. Endoscopic ultrasound-guided fine-needle aspiration (Fig. 4A and B) also revealed an SMT on the left side of the Ra portion of the rectum that extended from the submucosal layer beyond the serosal layer, and a lymph node sized $17 x 11 \mathrm{~mm}$ to the left of the Ra portion near the oral side $2 \mathrm{~cm}$ from the SMT. The pathological findings confirmed the SMT as adenocarcinoma with a metastatic lymph node. Local recurrence and lymph node recurrence of rectal cancer following radical EMR was diagnosed, as the SMT and lymph node were 
in proximity to the EMR scar and the pathological findings revealed adenocarcinoma in both the SMT and the lymph node. Laparoscopic ultra-low anterior resection, D3 lymph node dissection and a diverting ileostomy were performed. The operative time was $3 \mathrm{~h}$ and $41 \mathrm{~min}$, and the blood loss was $5 \mathrm{ml}$. The resected specimens (Fig. 5A and B) included an SMT lesion at the Ra portion of the rectum, and the periphery of the tumor exhibited a hard consistency from the submucosal layer extending beyond the serosal layer. The pathological findings (Fig. 6) were T3 (A/SS), ly0, v3, PN1b, pPM0, pDM0, pRM0 $(100 \mu \mathrm{m})$ and pN0 (0/15). XELOX therapy was administered for 6 months as adjuvant chemotherapy, and this was followed by chest and abdominal enhanced computed tomography scans every 6 months. The final follow-up appointment was performed during December 2017, and there has been no recurrence in the first 12 months after surgery.

\section{Discussion}

In recent years, EMR has been indicated for the treatment of superficial, early-stage colorectal cancer due to its minimal invasiveness and excellent results in terms of clinical outcome. The decision on the use of endoscopic resection is made in accordance with the JSCCR guidelines (1). Endoscopic resection is recommended in tumors with little possibility of lymph node metastasis and in tumors with a size and site (M or SM of $<1,000 \mu \mathrm{m}$ infiltration degree) permitting excision in bulk. The size and the visual system used to assess the tumor is not considered in the use of endoscopic resection. In addition, the criteria for additional resection of pT1 (SM) colon cancer that has already been resected (4) are also being investigated. It is desirable to add surgical resection in cases of a positive vertical stump. Furthermore, following histological examination of the obtained specimens, if the pathological findings reveal i) SM infiltration degree of $\geq 1,000 \mu \mathrm{m}$; ii) positive lymphovascular invasion; iii) poorly differentiated adenocarcinoma, signet ring cell carcinoma, or mucinous adenocarcinoma; or iv) conglobate (budding) lesion grade $2 / 3$ in cases of advanced infiltration, intestinal resection with lymph node dissection should be considered as an additional treatment. These criteria were applied to the 0-IIa lesion in the Ra portion of the rectum in the present case. The pathological findings at the time of EMR were tub1>tub2, the depth of invasion was SM1 $(420 \mu \mathrm{m})$, lymphatic invasion and venous invasion on hematoxylin and eosin (H\&E)-stained sections were both negative, and the resection margin was also negative. The tumor was resected in bulk. For these reasons, the JSCCR guidelines were followed, but local recurrence was discovered at 26 months after radical EMR. The pathological findings of the initial treating physician at our hospital were reviewed. Although the physician had based the diagnosis on H\&E staining alone, the presence of lymphatic invasion and venous invasion was diagnosed based on D2-40 staining and Victoria blue (VB)-H\&E staining (Fig. 7A and B) and the recurrence was diagnosed as ly1 and v1. Therefore, the accurate preoperative diagnosis was tub1>tub2, T1a (SM1, $420 \mu \mathrm{m})$, ly1 (D2-40) and v1 (VB-H\&E).

Cases of local recurrence following tumor removal by an endoscopic curative procedure according to the JSCCR guidelines are extremely rare. This is a valuable case: It is the 3rd reported case, to our knowledge, of local recurrence of T1a

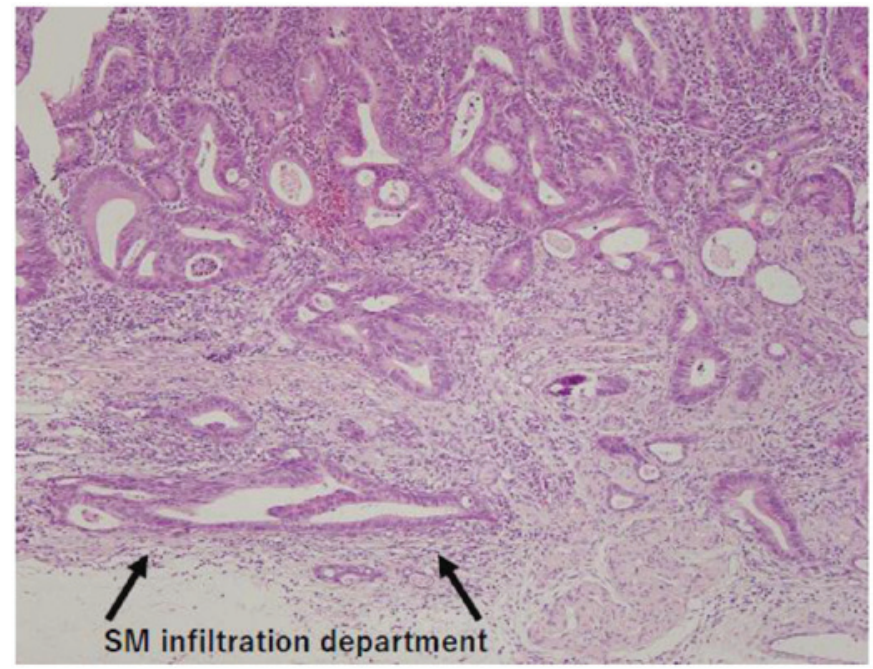

Figure 1. The pathological findings were tub1, T1a (SM1, $420 \mu \mathrm{m})$, ly0 and v0.

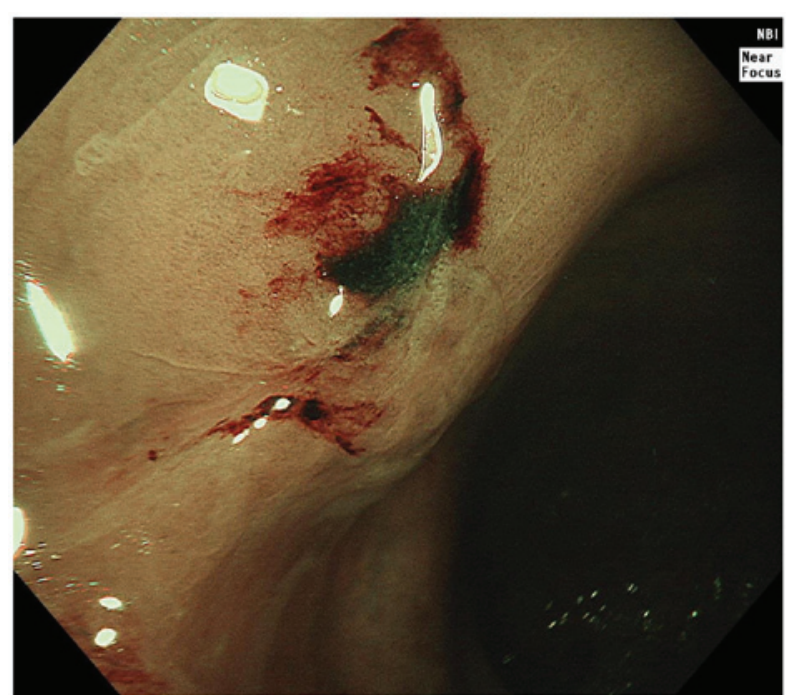

Figure 2. Colonoscopy findings. The SMT was located near the EMR scar in the Ra portion of the rectum. SMT, submucosal tumor; EMR, endoscopic mucosal resection.

rectal cancer following radical EMR in Japan, with the other two cases reported by Nobuharu et al (5) and Yosuke et al (6). In addition, the case reported by Nobuharu et al (5) suggested that, if the mucosal muscle layer is disrupted by the diffuse spread of cancer tissue, the risk of metastasis is high. The case reported by Yosuke et al was considered on a representative level, and the total percentage of cancer in the specimens was not examined. If the infiltrated portion is somewhat close to the resection margin, it may be possible for cross-end positivity or lymphovascular invasion positivity to be diagnosed if other sections of the total sample are considered.

The usefulness and limitations of using special staining methods, such as D2-40 and VB-H\&E, to determine lymphovascular invasion has been frequently reported in colon cancer. Lymphovascular invasion is the most important risk factor of metastasis (7), but diagnosis may be subjective depending on the pathologist (8). Akiko et al (9) reported that reproducibility of the diagnosis of lymphovascular invasion 


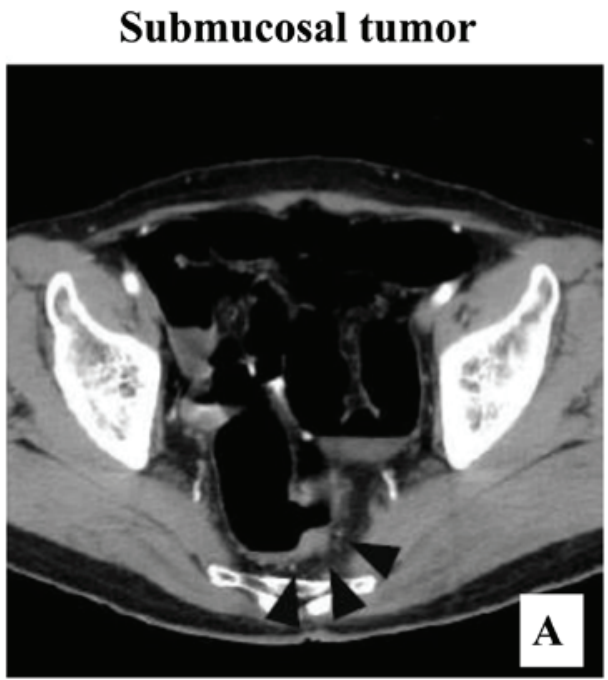

\section{Lymph node metastasis suspected}

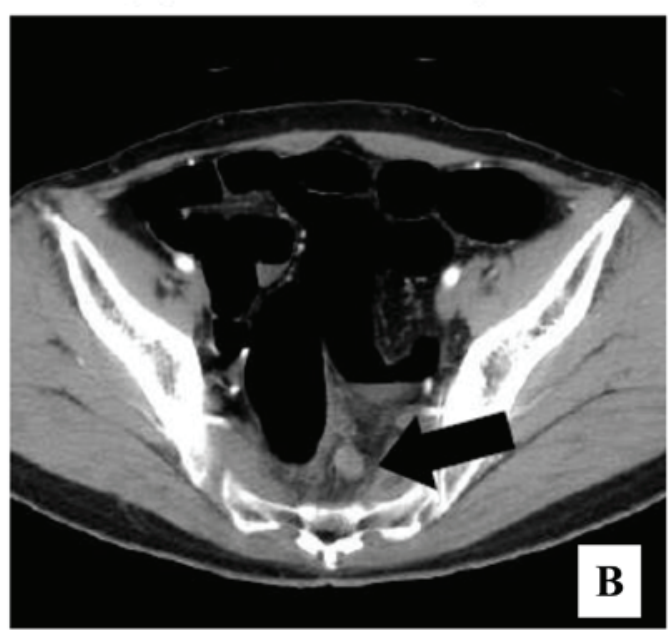

Figure 3. Abdominal enhanced computed tomography scan. (A) Infiltration of the full thickness of the left wall of the Ra portion of the rectum with limited extramural extension (arrowheads) and (B) a lymph node $10 \mathrm{~mm}$ in diameter (arrow) are shown.
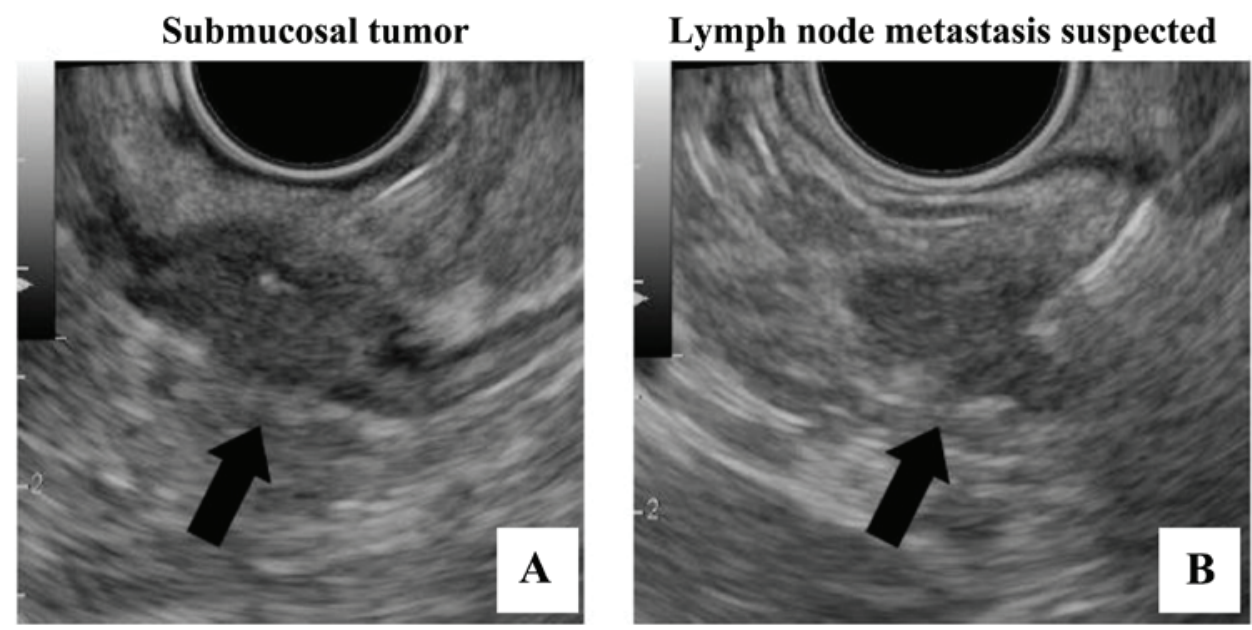

Figure 4. Endoscopic ultrasound-guided fine-needle aspiration. (A) An SMT on the left side of the Ra portion of the rectum extending from the submucosal layer to outside the serosal layer (arrow), and (B) a lymph node sized 17x11 mm (arrow) to the left of the Ra portion near the oral side $\sim 2 \mathrm{~cm}$ from the SMT tumor. SMT, submucosal tumor.
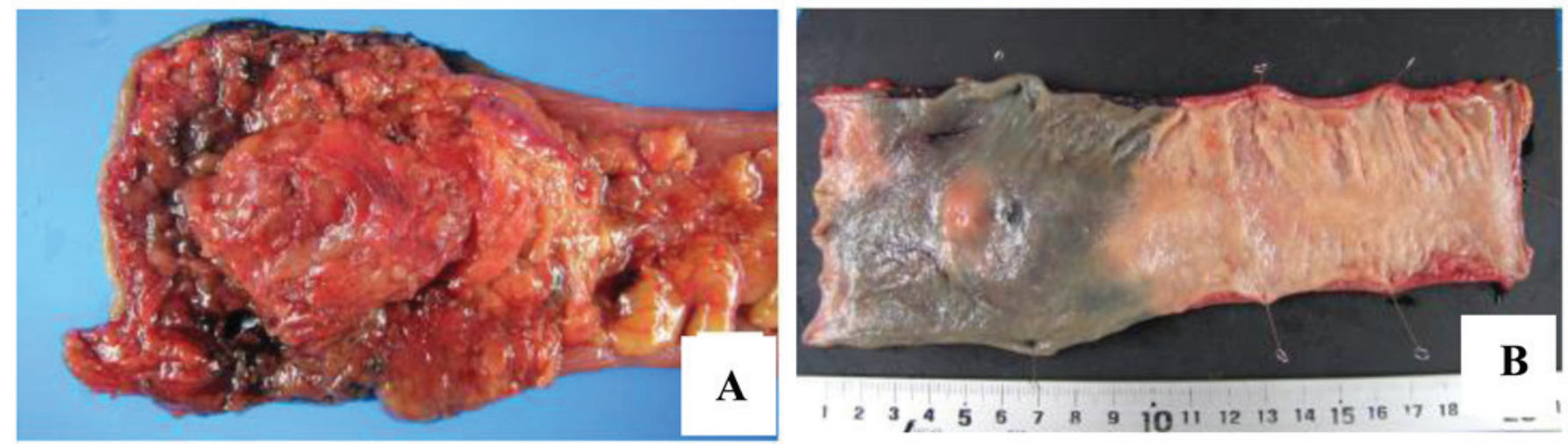

Figure 5. Macroscopic examination of the resected specimens from (A) the serosal side and (B) the mucosal side. An SMT lesion was identified at the Ra portion of the rectum: the periphery of the tumor exhibited a hard consistency extending from the submucosal layer to outside the serosal layer.

was problematic in specimens stained with $\mathrm{H} \& \mathrm{E}$, and it is better to consider the use of special staining methods to improve the diagnostic accuracy. Toshiki et al (10) reported on the results of special staining in 139 lesions in patients with SM cancer, 


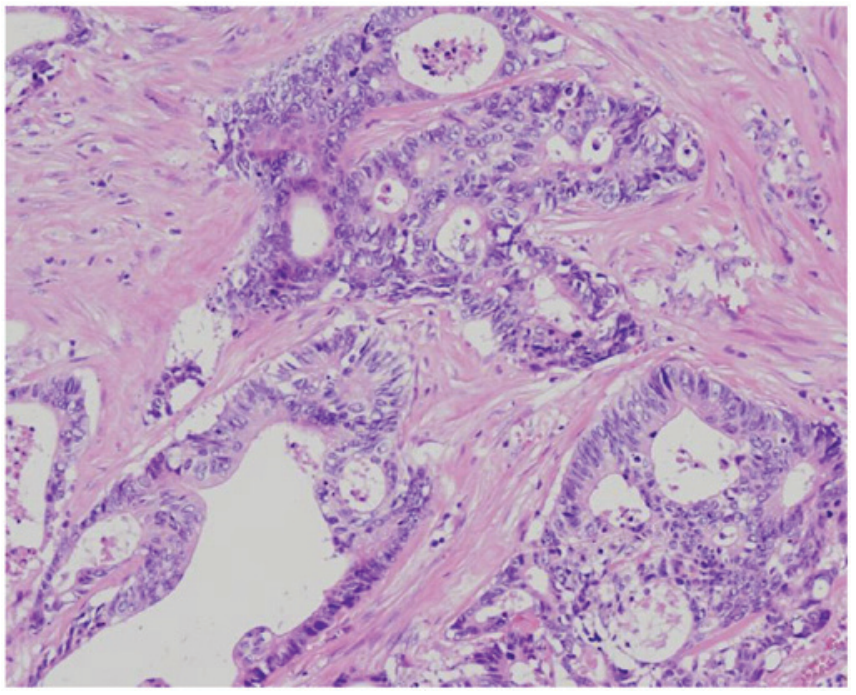

Figure 6. The pathological findings were T3 (A/SS), ly0, v3, PN1b, pPM0, pDM0, pRM0 $(100 \mu \mathrm{m})$, pN0 (0/15). Hematoxylin and eosin staining; magnification, $\mathrm{x} 400$.
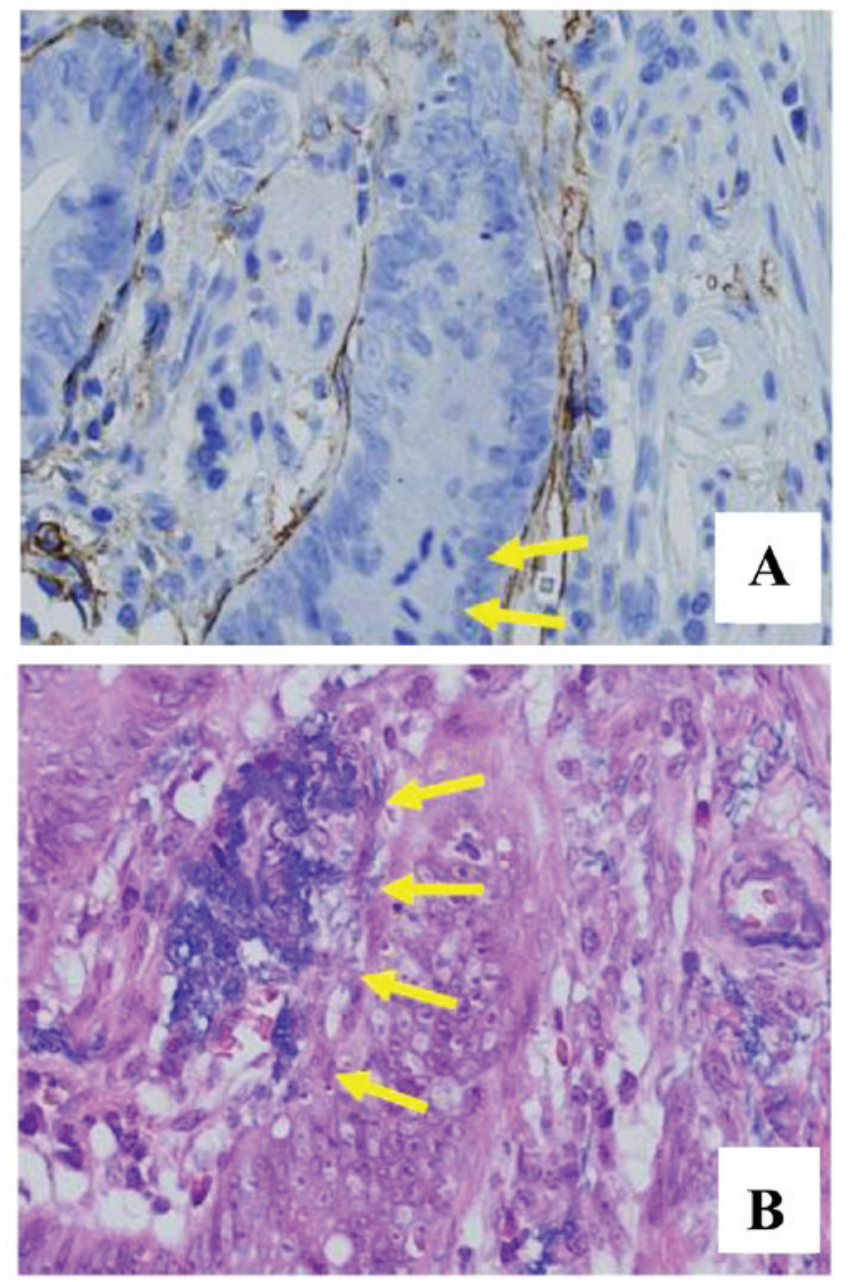

Figure 7. Pathological examination with special staining methods revealed (A) lymphatic invasion (D2-40 staining; magnification, $x 400$ ) and (B) venous invasion (VB-H\&E staining; magnification, $\mathrm{x} 400$ ), indicated bythe arrows.

and found that the diagnosis of lymph node metastasis by evaluating lymphovascular invasion based on H\&E staining exhibited a sensitivity of $75.0 \%$ and a specificity of 50.3\%, whereas the use of special staining exhibited a sensitivity of $93.8 \%$, a specificity of $46.0 \%$, a positive predictive value of $12.9 \%$ and a negative predictive value of $98.9 \%$. Thus, the improved sensitivity of special staining may be meaningful, as the absence of lymph node metastasis may be considered almost certain if the specimen is negative for lymphovascular invasion.

In summary, we herein reported an extremely rare case of cancer recurrence 26 months after the removal of a rectal tumor by EMR performed according to the JSCCR guidelines. The use of H\&E staining alone may make it difficult to diagnose lymphovascular invasion; therefore, the addition of special staining must be considered when the extent of lymphovascular invasion is unclear on H\&E staining.

\section{Acknowledgements}

Not applicable.

\section{Funding}

No funding was received.

\section{Availability of data and materials}

Not applicable.

\section{Authors' contributions}

NM and KM conceived and designed the study. NM, KM, TaT, ToT, SM, TS, JT, TI, HI, YT, KYa and KYo acquired the data. NM, KM, TaT, NS and TM analyzed and interpreted the data. NM and KM drafted the manuscript. NM, KM, TaT and KYo critically revised the manuscript for important intellectual content. KYo supervised the study and acquired the data. All the authors have read and approved the final version of this manuscript.

\section{Ethics approval and consent to participate}

Not applicable.

\section{Patient consent for publication}

Written informed consent was obtained from the patient for the publication of the case details and accompanying images.

\section{Competing interests}

KYo has received grants, personal fees and non-financial support from Chugai Pharmaceutical Co., Ltd., during the care of the reported patient; grants and personal fees from Taiho Pharmaceutical Co., Ltd., Pfizer Inc., Yakult Honsha Co., Ltd., and grants from Bristol-Myers Squibb and Kyowa Hakko Kirin Co., Ltd., outside the submitted work; honoraria from Taiho Pharmaceutical Co., Ltd., Pfizer Inc., Chugai Pharmaceutical Co., Ltd., Kyowa Hakko Kirin Co., Ltd., and Yakult Honsha Co., Ltd.; and had a consultant or advisory relationship with Taiho Pharmaceutical Co., Ltd. and La Roche, Ltd. TaT has 
received honoraria for lectures from Takeda Pharmaceutical Co., Ltd. All remaining authors declare that they have no competing interests to disclose.

\section{References}

1. Watanabe T, Muro K, Ajioka Y, Hashiguchi Y, Ito Y, Saito Y, Hamaguchi $\mathrm{T}$, Ishida $H$, Ishiguro $M$, Ishihara $S$, Kanemitsu $Y$, et al; Japanese Society for Cancer of the Colon and Rectum: Japanese Society for Cancer of the Colon and Rectum (JSCCR) guidelines 2016 for the treatment of colorectal cancer. Int J Clin Oncol 23: 1-34, 2018.

2. Yoshiro T, Yosuke F, Akiko C, Chika T, Sho S, Kenjiro M, et al: Progress of Endoscopic Treatment for Early Colorectal Cancers. J Jpn Soc Coloproctol 65: 800-807, 2012.

3. Masahiro T, Yasumasa N, Vikram B, Shinya K, Tsutomu T, Nobumasa $\mathrm{M}$, et al. Comparison of endoscopic submucosal dissection and endoscopic mucosal resection for large colorectal tumors. Eur J Gastroenterol Hepatol 23: 1042-1049, 2011.

4. Tajika M, Niwa Y, Bhatia V, Kondo S, Tanaka T, Mizuno N Hara K, Hijioka S, Imaoka H, Ogura T, et al: Comparison of endoscopic submucosal dissection and endoscopic mucosal resection for large colorectal tumors. Eur J Gastroenterol Hepatol 23: 1042-1049, 2011.

5. Nobuharu T, Masahiro I, Kiyonori K, Yasushi S, Yusuke S, Hironori Y, et al: Surveillance of colorectal cancer after endoscopic treatment. Colon Dis 2007: 112-120, 2007 (In Japanese).
6. Yosuke K, Yoshin T, Saikyo M, Takahiro T, Koichiro T, Koji S, et al: A resected case of local progressive sigmoid colon carcinoma after radial endoscopic mucosal resection for SM1 carcinoma. Clin Res 90: 127-305, 2013 (In Japanese).

7. Hirohiko K, Masahiro I, Jimi M and Mitsuyoshi U: Risk Factor for Lymph Node Metastasis of Colorectal Carcinomas with Submucosal Invasion. Tokyo Jikeikai Med J 124: 113-126, 2009.

8. Hirokazu T, Yuri F, Shigeki S, Tadakazu S and Ryoji K: Vascular Infiltration of Submucosal Invasive Colorectal Cancer. Stomach Intestine Tokyo 44: 1241-1248, 2009.

9. Akiko M, Ryoji K, Hirokazu T and Shigeki S: Special Stains Useful for Diagnosis of Colon Tumors. Stomach Intestine Tokyo 45: 699-704, 2010

10. Toshiki N, Shoichi S, Hironori I, Hirohiko K, Jimi M, Hiroyuki A, et al: Risk Factors for Lymph Node Metastasis of Submucosal Invasive Colon Cancer-Emphasis on Detection of Vessel Permeation Using Special Stains. Stomach Intestine Tokyo 46: 1459-1468, 2011.

This work is licensed under a Creative Commons Attribution-NonCommercial-NoDerivatives 4.0 International (CC BY-NC-ND 4.0) License. 